\title{
RESEARCH
}

Open Access

\section{Should ice application be replaced with neurocryostimulation for the treatment of acute lateral ankle sprains? A randomized clinical trial}

\author{
Jean Tittley ${ }^{1,2}$, Luc J. Hébert ${ }^{1,2,3}$ and Jean-Sébastien Roy ${ }^{1,2^{*}}$ (D)
}

\begin{abstract}
Study design: Single-blind parallel group randomized clinical trial.

Objectives: To compare the effects of neurocryostimulation (NCS) with those of traditional ice application on functional recovery, pain, edema and ankle dorsiflexion range of motion (ROM) in individuals receiving physiotherapy treatments for acute lateral ankle sprains (LAS).

Background: Ankle sprain is a very common injury and its management is often costly, with important short- and long-term impacts on individuals and society. As new methods of therapy using cold (cryotherapy) are emerging for the treatment of musculoskeletal conditions, little evidence exists to support their use. NCS, which provokes a rapid cooling of the skin with the liberation of pressured $\mathrm{CO}^{2}$, is a method believed to accelerate the resorption of edema and recovery in the case of traumatic injuries.

Methods: Forty-one participants with acute LAS were randomly assigned either to a group that received in-clinic physiotherapy treatments and NCS (experimental NCS group, $n=20$ ), or to a group that received the same in-clinic physiotherapy treatments and traditional ice application (comparison ice group, $n=21$ ). Primary (Lower Extremity Functional Scale - LEFS) and secondary (visual analog scale for pain intensity at rest and during usual activities in the last $48 \mathrm{~h}$, Figure of Eight measurement of edema, and weight bearing lunge for ankle dorsiflexion range of motion) outcomes were evaluated at baseline (T0), after one week (T1), two weeks (T2), four weeks (T4) and finally, after six weeks (T6). The effects of interventions were assessed using two-way ANOVA-type Nonparametric Analysis for Longitudinal Data (nparLD).
\end{abstract}

Results: No significant group-time interaction or group effect was observed for all outcomes ( $0.995 \geq p \geq 0.057)$ following the intervention. Large time effects were however observed for all outcomes $(p<0.0001)$.

(Continued on next page)

\footnotetext{
* Correspondence: Jean-Sebastien.Roy@fmed.ulaval.ca

'Department of Rehabilitation, Faculty of Medicine, Université Laval, Quebec City, Quebec, Canada

${ }^{2}$ Center for Interdisciplinary Research in Rehabilitation and Social Integration, Quebec Rehabilitation Institute, Quebec City, Quebec, Canada

Full list of author information is available at the end of the article
}

(c) The Author(s). 2020 Open Access This article is licensed under a Creative Commons Attribution 4.0 International License, which permits use, sharing, adaptation, distribution and reproduction in any medium or format, as long as you give appropriate credit to the original author(s) and the source, provide a link to the Creative Commons licence, and indicate if changes were made. The images or other third party material in this article are included in the article's Creative Commons licence, unless indicated otherwise in a credit line to the material. If material is not included in the article's Creative Commons licence and your intended use is not permitted by statutory regulation or exceeds the permitted use, you will need to obtain permission directly from the copyright holder. To view a copy of this licence, visit http://creativecommons.org/licenses/by/4.0/ The Creative Commons Public Domain Dedication waiver (http://creativecommons.org/publicdomain/zero/1.0/) applies to the data made available in this article, unless otherwise stated in a credit line to the data. 


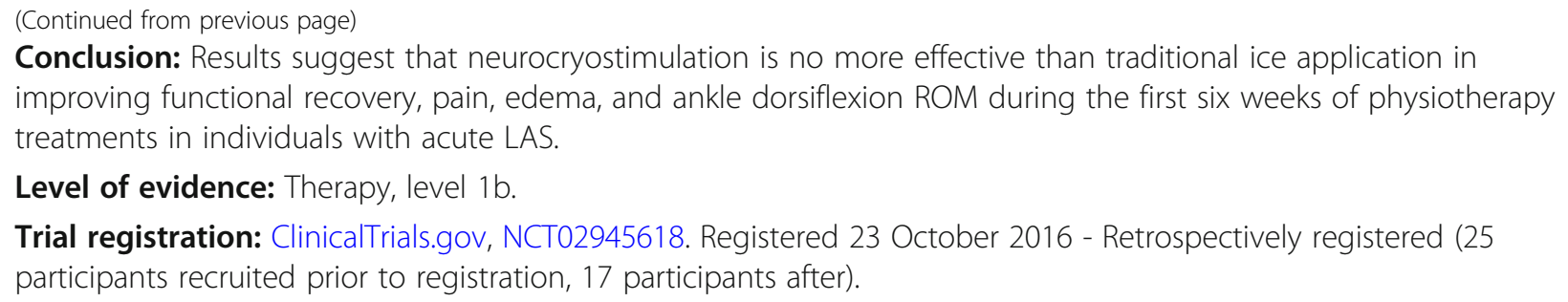

Keywords: Lateral ankle sprain, Neurocryostimulation, Cryotherapy, Rehabilitation

\section{Background}

Ankle sprains represent up to $25 \%$ of all musculoskeletal injuries, and half of them are sports-related [1]. Sprain can be defined as the injury that occurs when a tensile force causes stretching, partial tearing or complete tearing of a ligament [2]. Lateral sprains are the most frequent type of ankle sprain, occurring at least two times more frequently than all other ankle sprains combined [3]. Considering that 20 to $50 \%$ of individuals who suffer ankle sprains develop chronic pain or chronic ankle instability, the prognosis is less than optimistic [4]. These realities impel constant efforts to identify optimal treatment plans.

The most widely-used treatment for acute-phase ankle sprains is summarized by the RICE acronym (Rest, Ice, Compression, Elevation) [5]. However, the rationale behind the use of RICE, as well as for each of its individual components, is largely based on a very limited evidence, consisting mainly of low-quality clinical trials and laboratory studies on healthy subjects or on animals [6-8]. Systematic reviews on acute ankle sprain treatments report evidence supporting the use of early mobilization (weight-bearing as tolerated and mobilization exercises), analgesic drugs, splints or braces, as well as manual therapy [3, 8-11]. However, scientific evidence on the effect of cryotherapy specifically is limited and inconsistent. That said, one systematic review concluded that cryotherapy is effective in decreasing pain in the short term (over a one-week period) in acute-phase of soft tissue injuries [12]. In addition, a 2018 clinical guideline reports that there is no evidence that cryotherapy alone is effective in improving pain, function, or swelling in acute lateral ankle sprains (LAS), but that it improves ankle function in the short term when combined with exercises [8]. Moreover, no consensus has been reached concerning the best cryotherapy protocols or application methods $[12,13]$.

Neurocryostimulation (NCS), also known as gaseous hyperbaric cryotherapy, is a treatment modality in which compressed carbonic gas is projected from a medical gun onto a patient's skin at high speed. A rapid and considerable drop in skin temperature ensues, causing thermal shock, which, in healthy subjects, is a swift systemic response resulting in cutaneous vasoconstriction and increased blood pressure [14, 15]. These effects on the autonomic nervous system have been proposed to accelerate edema resorption, thus quickening recovery and healing following soft tissue injuries [16]. Other suggested effects of NCS include analgesia (by nociceptor inhibition), decreased inflammation (by suppression of enzyme activity), vasomotor effects (by profound vasodilation after 20 to $30 \mathrm{~s}$ of application), and muscle relaxation (resulting from a myostatic reflex in the medulla) [16]. However, these proposed effects remain highly speculative, as only few studies have evaluated the effect of NCS in symptomatic populations, and most of them present important methodological shortcomings in either the experimental design (no comparison group) [16] or the NCS application protocol (attaining suboptimal temperatures) [17].

The objective of this study was to evaluate the effects of neurocryostimulation (NCS) in comparison with those of traditional ice application on functional recovery, pain, edema and ankle dorsiflexion range of motion (ROM) in individuals receiving physiotherapy treatments for acute LAS, using a single-blind randomized clinical trial. The hypothesis was that participants receiving NCS treatment would improve more rapidly for all measured variables than those treated with ice. This RCT was registered on ClinicalTrials.gov (NCT02945618).

\section{Methods \\ Participants}

Potential participants were recruited using a university community's electronic mailing list (Université Laval, Quebec City) and at the university's physiotherapy clinic. A physiotherapist in charge of recruitment and evaluations contacted all interested individuals for prescreening and scheduled a first appointment at the clinic with potential participants to confirm the following criteria: 1) had experienced a moderate to high grade LAS in the $72 \mathrm{~h}$ preceding recruitment, 2) were aged 18 and above, and 3) were available to participate in eight physiotherapy treatments and five evaluation sessions over a 6-week period. Potential participants were excluded if they: 1) had experienced a previous LAS in the 
12 months preceding recruitment, 2) had ever suffered a foot or ankle fracture, 3) had residual signs or symptoms of a previous foot or ankle injury, 4) had any lacerations, wounds or other conditions affecting skin integrity at the treatment site, 5) presented any contraindications to cryotherapy treatment (such as peripheral vascular disease, Raynaud's syndrome, cold urticaria, or cryoglobulinemia) or altered skin sensitivity. Potential participants were also excluded if the principal injury, as diagnosed in the baseline evaluation, was not to the lateral ankle ligaments, but rather to the distal tibiofibular syndesmosis (assessed with the squeeze test, the dorsiflexionexternal rotation test, and palpation) [18-20]. For the purpose of this study, a moderate to high grade LAS diagnosis was confirmed with the presence of all of the followings: 1) an history of traumatic onset, 2) pain and limping (or incapacity) at walking, 3) visible edema on the lateral aspect of the ankle, and finally, 4) pain at palpation of the anterior talofibular ligament or the calcaneofibular ligament, and/or an augmented range of movement in at least one test among the anterior drawer, the anterior talofibular ligament stress test and the calcaneofibular ligament stress test. As there is no clear consensus on how to diagnose and classify LAS, these criteria were chosen to reflect current clinical guidelines $[1,5,6,21,22]$, Potential participants presenting with positive Ottawa ankle rules who had not undergone radiography evaluation were sent for medical examination in order to exclude possible fractures [23]. The sectorial health sciences research ethics committee of Université Laval approved this study (\#2015-053).

\section{Study design}

A single-blind, parallel group randomized clinical trial with blinded evaluator was realized (see Fig. 1. Study Design). Following the baseline evaluation, participants were randomly assigned to either the NCS (experimental: standardized rehabilitation program $+\mathrm{NCS}$ ) or the ice (comparison: same standardized rehabilitation program + ice application) group. Both groups received standardized physiotherapy treatments, consisting of eight treatments over a four-week period (three treatments per week for two weeks, followed by one treatment per week for two weeks). They were evaluated five times in total: at baseline (T0), one week (T1), two weeks (T2), four weeks (T4) and six weeks (T6). Sociodemographic data were collected at T0. Functional capacity (the primary outcome), pain, ankle dorsiflexion ROM and edema measurements were taken at each of the five evaluation sessions.

\section{Randomization and blinding}

A randomization list, generated by an independent researcher not involved in data collection, was established prior to initiation of the study using a random number generator (block randomization; block size of 4,6 or 8). Randomization was stratified according to sex (male/female). Allocation was concealed in sealed and opaque envelopes sequentially numbered. After validation of the eligibility criteria, the baseline assessment was immediately conducted and then an independent researcher opened the sealed envelope and informed the treating physiotherapist of the group assignment. Participants were unaware of the treatment provided to the participants in the other group, nor that the cryotherapy was the central element of this RCT. Before every evaluation session, participants were reminded not to share their group assignment with the evaluator. All these evaluations took place in a closed room, separate from the treating area of the physiotherapy clinic. To assess blinding effectiveness, the evaluator answered the following question at the week-6 evaluation: "In your opinion,

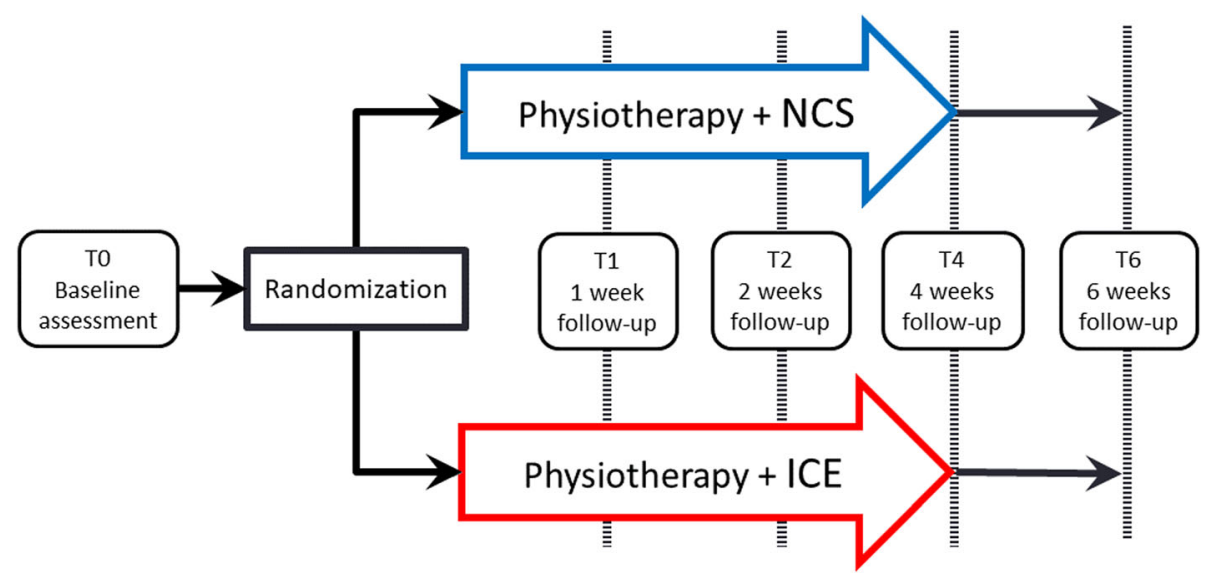

Fig. 1 Study Design. NCS: neurocryostimulation 
which intervention did this participant receive?" The possible answers were: NCS (experimental group); ice application (comparison group); I have no idea.

\section{Outcome measures}

The primary outcome was functional capacity, as measured by the Lower Extremity Functional Scale (LEFS). The LEFS is a self-administered questionnaire consisting of 20 items [24]. It has been validated in individuals with lateral ankle sprain [25], and its reliability, construct validity and responsiveness have all been demonstrated [24-27]. The validated French Canadian version of the questionnaire was used for the current study [28, 29]. The minimal detectable change (MDC) and the minimal clinically important difference (MCID) are both 6 points [27].

Secondary dependent variables included pain intensity, ankle edema, and dorsiflexion ROM. Two visual analog scales (VAS) were filled to evaluate perceived pain intensity at rest and during usual activities in the last $48 \mathrm{~h}$ $(10 \mathrm{~cm}$ scale varying from 0 ["no pain"] to 10 ["worst pain imaginable"]) [30]. The reliability of this scale has been demonstrated, and its MCID is $1.3 \mathrm{~cm}$ in individuals with acute pain [31]. The Figure of Eight method measures ankle size and edema using precise anatomical landmarks, and has been found to be reliable and valid in populations with ankle sprains or other injuries of the ankle or foot [32-34]. The minimal detectable change $\left(\mathrm{MDC}_{95 \%}\right)$ is $0.96 \mathrm{~cm}$ for swollen ankles [35]. Finally, the employed dorsiflexion range of motion (ROM) measurement, the Weight-Bearing Lunge Test (WBLT), is standardized, simple, fast and reliable. The distance between the big toe and a wall is measured in a forward lunge in weight-bearing (with the knee touching the wall). Its MDC has been established at $1.6 \mathrm{~cm}$ (inter-evaluator) or $1.9 \mathrm{~cm}$ (intra-evaluator) [36].

\section{Interventions}

Both groups received the same rehabilitation program. The only between-group difference was the application of ice in the comparison group and the use of NCS in the experimental group. Three physiotherapists, accustomed to working with individuals with acute ankle injuries, administered the treatments in an outpatient physiotherapy clinic on the university campus. All three physiotherapists treated participants within both allocation groups. They were properly trained in the study protocol and rehabilitation program, and they practiced the cryotherapy procedures often enough to be at ease and effective before the onset of the study. While they were asked to respect their own clinical judgment for the elaboration of their treatment plan, they were required to prescribe and teach stretching, strengthening and balance exercises and to perform joint mobilizations. They were also advised to use a compression and support modality such as a laced brace, taping or elastic bandaging. On the contrary, they were not permitted to use ultrasound, electrotherapy (such as transcutaneous electrical nerve stimulation or interferential current), heat application or dry needling modalities. This rehabilitation program follows current clinical guidelines $[1,5,6,37]$. In-clinic sessions therefore consisted mainly of education on the injury and the healing process, teaching and practicing exercises, joint mobilization and soft tissue techniques, and management of compression and support methods. The parameters and details of each of these interventions were adjusted at the physiotherapist's discretion according to each participant's condition and progress.

In both groups, cryotherapy treatments were administered at the end of each physiotherapy session. For the NCS group, NCS was applied following the manufacturer's instructions (Cryofos Medical GmbH, Germany), which is similar to application methods used in other studies $[16,17]$. Slow sweeping motions of the medical gun were used over zones approximately the size of a credit card, for a maximum of 2 min, until a skin temperature of $4^{\circ}$ Celsius was reached. Skin temperature was measured constantly by a thermometer contained within the medical gun, and the desired temperature of $4^{\circ}$ Celsius was usually attained within $30 \mathrm{~s}$ of application. This procedure was applied to two distinct zones in order to cover the full surface area of the lateral ankle and was then repeated on the medial ankle and posterior to the knee, due to the presence of lymphatic ganglions at the latter site. For the ice group, the cryotherapy consisted of two bags of crushed ice (each approximately $25 \times 25 \mathrm{~cm}$ ) applied around the injured ankle in order to cover the joint entirely for 15 min, with the legs elevated. Skin temperature of the lateral treated ankle was measured before and immediately following each cryotherapy treatment in both groups, using the thermometer contained within the NCS medical gun.

\section{Sample size calculation}

The required sample size was established at 36 subjects, 18 in each group, based on a standard deviation of 12.5 points and a MCID of 6.3 points on the primary outcome (LEFS questionnaire) [26] as well as a $15 \%$ loss to follow-up, using G*Power 3.1.7 software. Alpha and beta levels were set at $\alpha=0.05, \beta=0.80$, with effect sizes of 0.80 .

\section{Statistical analyses}

Baseline sociodemographic data were compared between the groups using independent $\mathrm{t}$-tests and chi-squared tests. Normality of the distributions was verified for each 
of the variables. Nonparametric Analysis for Longitudinal Data (nparLD Package 2.1, R-software, v.3.3.3) for repeated measures were used since distributions were normal at baseline (as there was a relatively wide range of scores for every single outcome) and gamma at the latest assessment times (as most participants improved close to optimal values). The nparLD is a procedure that manages a change of distribution between groups and measurement times [38]. A two-way (2-Groups [NCS, ice] $\times 5$-Time [T0, T1, T2, T4 and T6]) nparLD was used to compare NCS and ice effects on each of the dependent variables. Intention-to-treat procedures were followed, and on the rare occasions that data were missing, the result from the previous evaluation was used (Last-Observation-carried-Forward method). The $\alpha$ criterion was always set at $5 \%$.

\section{Results}

Fifty-seven potential participants were evaluated between June 2015 and October 2017. Of these, 42 met the inclusion criteria and were included in the study (see Fig. 2.
Flowchart of Participants). Fifteen were excluded at this point for the following reasons: grade 1 sprain (neither pain nor limping in walking, $n=10$ ), tibio-fibular syndesmosis $(n=3)$ or mid-foot $(n=1)$ as the primary site of injury, and presence of chronic knee pain limiting daily activities $(n=1)$. One participant, who was included in the study and randomized to the NCS group, still presented with major functional limitations at 6 weeks and was found to have ankle intra-articular osteochondral lesions on magnetic resonance imaging (MRI). This participant was excluded from the final analyses. No adverse event was reported during the course of the study among participants.

Average delays between the occurrence of the injury and the initial evaluation, and between the injury and the first treatment, were 2.2 (standard deviation [SD] 1.0) and 2.8 (SD 1.1) days, respectively. All subjects attended all eight treatments. Only one participant missed an evaluation (the 6-week follow-up, NCS group) and one other missed the edema and dorsiflexion ROM assessment at the 4-week and 6-week follow-ups (filled

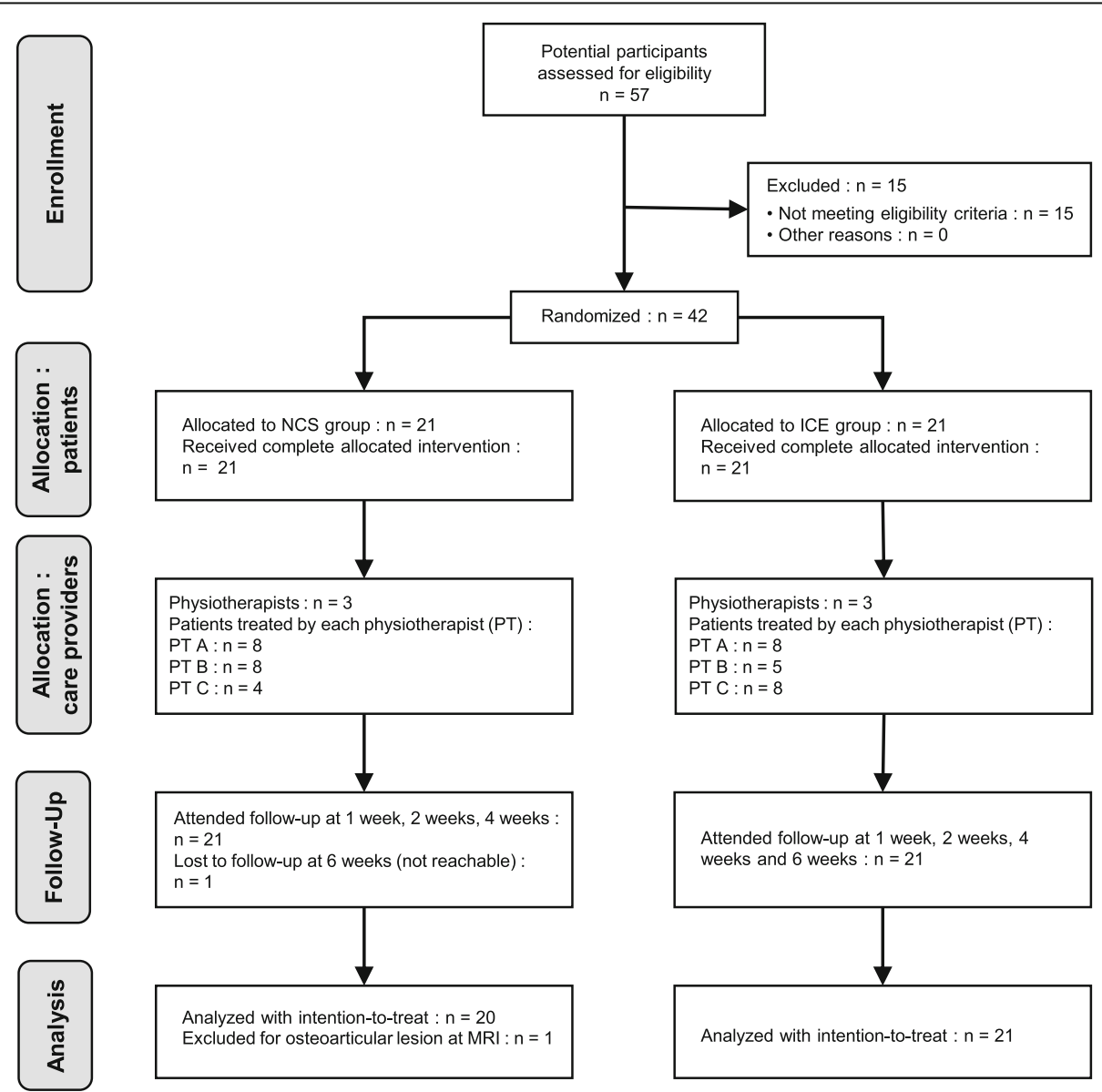

Fig. 2 Flowchart of Participants. MRI: magnetic resonance imaging; NCS: neurocryostimulation; PT: physiotherapist 
the LEFS and pain VAS remotely). Of the total outcome data, 13 measures were missing out of 1025 , with only one missing for our primary outcome. The evaluator indicated that he remained unaware of group assignment for all participants throughout the study.

Participants' baseline characteristics are presented in Table 1. No differences between the groups were found for all measured variables. In the NCS group, the average (mean of all eight sessions) decrease in skin temperature following cryotherapy was $23.7^{\circ} \mathrm{C}$ (SD 4.6), compared to $15.3^{\circ} \mathrm{C}$ (SD 5.0) in the ice group. Average (mean of all eight sessions) postcryotherapy skin temperatures reached were $8.4{ }^{\circ} \mathrm{C}$ (SD 4.1) in the NCS group, and $16.9^{\circ} \mathrm{C}$ (SD 5.0) in the ice group.

Table 2 presents the $p$-values of the ANOVA-type analysis (nparLD). For the primary outcome, the LEFS, no group $\mathrm{x}$ time interaction was found $(p=$ 0.73). Rapid improvement was observed in both groups, particularly during the first two weeks of treatment, with a large time effect $(p<0.001)$. In both the NCS and ice groups, LEFS mean score change was greater than the MCID between T0 and $\mathrm{T} 1, \mathrm{~T} 1$ and $\mathrm{T} 2$, and $\mathrm{T} 2$ and $\mathrm{T} 4$. No group $\mathrm{x}$ time interaction was demonstrated for the secondary outcomes of pain intensity at rest and during usual activities $(p=0.06$ and 0.65 , respectively), dorsiflexion $\operatorname{ROM}(p=0.77)$ and edema $(p=0.24)$, and substantial time effects were observed for all of these variables $(p<0.001)$. Table 3 presents the marginal estimated means by group at all measure times and Table 4 presents scores' changes over time, for all outcomes. Figures 3, 4 and 5 shows graphically the evolution by group for the LEFS, pain at rest and pain during usual activities mean scores, respectively.

\section{Discussion}

This RCT compared the effect of two cryotherapy interventions, namely neurocryostimulation and traditional ice application, on recovery in individuals suffering from acute LAS receiving physiotherapy treatments. Results indicated no difference between ice application and NCS throughout the 6-week follow-up period for any of the outcomes. Analyses demonstrated large time effects for all studied variables, as all participants improved rapidly regardless of treatment modality. There are several possible explanations for the comparable results seen between the groups: 1) the interventions are equally effective, 2) neither of the interventions is more effective than the mere passage of time, or 3) possible betweengroup differences were hidden by a stronger effect of the global physiotherapy treatment, or by the participants' rapid improvement, which is typical of this type of injury. Potential differences between NCS and ice application effects could thus be better detected in populations with conditions or injuries that typically progress more slowly.

Few clinical studies measuring the effect of NCS in populations presenting with musculoskeletal injuries have been published in peer-reviewed journals, and none has treated ankle sprains specifically. When studying the effect of NCS in the elderly, Chatap et al. concluded that NCS decreases acute and chronic pain of various etiologies; [16 ] however, they did not have a control or comparison group. In contrast, in a pilot study, Richer et al. failed to find differences when comparing the effects of NCS combined with manual therapy to those of manual therapy alone on pain, function and pain-free grip strength in patients with chronic lateral epicondylitis [39]. Similarly, in a RCT, Demoulin et al. did not find any between-group differences when comparing the effects of NCS and two other types of cryotherapy (gel

Table 1 Participants' baseline characteristics

\begin{tabular}{|c|c|c|c|}
\hline & & NCS group $(n=20)$ & Ice group $(n=21)$ \\
\hline \multicolumn{2}{|l|}{ Age, years, $\bar{X} \pm S D$} & $26.9 \pm 9.1$ & $28.3 \pm 9.8$ \\
\hline \multirow[t]{2}{*}{ Gender, \# (\% of allocation group) } & Female & $10(50 \%)$ & $10(48 \%)$ \\
\hline & Male & $10(50 \%)$ & $11(52 \%)$ \\
\hline \multicolumn{2}{|l|}{ Weight, $\mathrm{kg}, \bar{X} \pm \mathrm{SD}$} & $73.8 \pm 12.9$ & $72.1 \pm 14.4$ \\
\hline \multicolumn{2}{|l|}{ Height, $\mathrm{cm}, \bar{X} \pm S D$} & $172.4 \pm 9.3$ & $171.9 \pm 9.4$ \\
\hline \multicolumn{2}{|l|}{ Body mass index, $\mathrm{kg} / \mathrm{m}^{2}, \bar{x} \pm \mathrm{SD}$} & $24.8 \pm 3.8$ & $24.5 \pm 5.6$ \\
\hline \multirow[t]{2}{*}{ Number of previous ankle sprains, $\bar{X} \pm S D$} & Same ankle & $0.9 \pm 1.4$ & $1.0 \pm 1.4$ \\
\hline & Any ankle & $1.9 \pm 2.8$ & $1.5 \pm 2.1$ \\
\hline \multicolumn{2}{|c|}{ Physical activity, mean hours/week last 12 months, $\bar{X} \pm$ SD } & $10.6 \pm 8.2$ & $7.1 \pm 4.5$ \\
\hline \multicolumn{2}{|l|}{ Days from injury to initial assessment, $\bar{X} \pm S D$} & $2.4 \pm 0.9$ & $2.0 \pm 1.1$ \\
\hline \multicolumn{2}{|l|}{ Days from injury to 1 st treatment, $\bar{X} \pm S D$} & $3.0 \pm 1.1$ & $2.5 \pm 1.1$ \\
\hline
\end{tabular}

NCS neurocryostimulation, $S D$ standard deviation, $\bar{X}$ mean. There was no statistical difference between the two groups for all variables ( $\mathrm{P} \geq 0.05$; independent $\mathrm{t}-$ tests or Chi-squared tests). 
Table 2 Results ( $p$-values) of ANOVA-type analysis (nparLD) for the intention-to-treat analysis

\begin{tabular}{llll}
\hline & Group effect & Time effect & Group X Time interaction \\
\hline Functional capacity (LEFS) & 0.404 & $<0.0001$ & 0.727 \\
Pain at rest & 0.390 & $<0.0001$ & 0.057 \\
Pain during usual activities & 0.995 & $<0.0001$ & 0.648 \\
Oedema (Figure-of-8) & 0.563 & $<0.0001$ & 0.242 \\
Dorsiflexion ROM (WBLT) & 0.408 & $<0.0001$ & 0.766
\end{tabular}

LEFS Lower Extremity Functional Scale, nparLD non-parametric longitudinal data, ROM range of motion, WBLT Weight Bearing Lunge Test.

Table 3 Group marginal estimated means for all outcomes. Data expressed as mean \pm standard error of the mean (SEM)

\begin{tabular}{lll}
\hline & $\begin{array}{l}\text { NCS } \\
\text { (experimental } \\
\text { group, } \boldsymbol{n = 2 0 )}\end{array}$ & $\begin{array}{l}\text { Ice } \\
\text { (comparison } \\
\text { group, } \boldsymbol{n}=\mathbf{2 1} \text { ) }\end{array}$ \\
\hline Functional capacity (LEFS scores, $\mathbf{0 - 8 0 )}$ & \\
Baseline & $29.6 \pm 1.7$ & $34.7 \pm 2.5$ \\
1 week & $51.5 \pm 2.9$ & $52.2 \pm 2.6$ \\
2 weeks & $62.4 \pm 2.3$ & $64.7 \pm 2.0$ \\
4 weeks & $72.2 \pm 1.9$ & $73.0 \pm 1.1$ \\
6 weeks & $74.7 \pm 1.3$ & $76.3 \pm 0.8$ \\
Pain at rest (VAS, 0-10) & \\
Baseline & $1.9 \pm 0.4$ & $2.2 \pm 0.4$ \\
1 week & $1.5 \pm 0.4$ & $0.9 \pm 0.2$ \\
2 weeks & $0.8 \pm 0.3$ & $0.3 \pm 0.1$ \\
4 weeks & $0.2 \pm 0.2$ & $0.1 \pm 0.1$ \\
6 weeks & $0.1 \pm 0.1$ & $0.1 \pm 0.0$
\end{tabular}

Pain during usual activities (VAS, 0-10)

$\begin{array}{lll}\text { Baseline } & 4.5 \pm 0.5 & 4.6 \pm 0.4 \\ 1 \text { week } & 2.4 \pm 0.4 & 2.3 \pm 0.4 \\ 2 \text { weeks } & 1.7 \pm 0.4 & 1.2 \pm 0.2 \\ 4 \text { weeks } & 0.7 \pm 0.3 & 0.5 \pm 0.2 \\ 6 \text { weeks } & 0.4 \pm 0.2 & 0.2 \pm 0.1\end{array}$

Oedema (Figure-of-8, cm)

$\begin{array}{lll}\text { Baseline } & 53.7 \pm 0.7 & 52.7 \pm 0.8 \\ 1 \text { week } & 53.0 \pm 0.7 & 52.0 \pm 0.8 \\ 2 \text { weeks } & 52.6 \pm 0.7 & 52.1 \pm 0.8 \\ 4 \text { weeks } & 52.4 \pm 0.6 & 51.7 \pm 0.7 \\ 6 \text { weeks } & 52.5 \pm 0.7 & 51.9 \pm 0.8\end{array}$

Dorsiflexion ROM (WBLT, cm)

$\begin{array}{lll}\text { Baseline } & 5.5 \pm 0.8 & 5.9 \pm 1.0 \\ 1 \text { week } & 7.9 \pm 0.9 & 9.4 \pm 0.5 \\ 2 \text { weeks } & 9.6 \pm 0.7 & 10.6 \pm 0.5 \\ 4 \text { weeks } & 10.7 \pm 0.5 & 11.7 \pm 0.5 \\ 6 \text { weeks } & 11.2 \pm 0.5 & 11.7 \pm 0.6\end{array}$

LEFS Lower Extremity Functional Scale, NCS neurocryostimulation, ROM range of motion, VAS visual analog scale, WBLT Weight-Bearing Lunge Test.
Table 4 Outcomes changes over time compared to baseline values throughout treatment (marginal estimated mean improvements), by group

\begin{tabular}{|c|c|c|c|}
\hline & \multicolumn{2}{|c|}{ Mean score change $(95 \% \mathrm{Cl})$} & \multirow{2}{*}{$\begin{array}{l}\text { Time } \\
\text { main } \\
\text { effect } \\
(\mathrm{RTE})^{\mathrm{a}} \\
(n= \\
41)\end{array}$} \\
\hline & $\begin{array}{l}\text { NCS } \\
\text { (experimental } \\
\text { group, } n=20 \text { ) }\end{array}$ & $\begin{array}{l}\text { Ice } \\
\text { (comparison } \\
\text { group, } n=21 \text { ) }\end{array}$ & \\
\hline \multicolumn{4}{|c|}{ Functional capacity (LEFS scores, 0-80) } \\
\hline 1 week & $22.0(13.6$ to 30.3$)$ & $17.6(10.2$ to 25.0$)$ & 0.340 \\
\hline 2 weeks & 32.8 (26.0 to 39.6$)$ & 30.1 (22.4 to 37.7$)$ & 0.514 \\
\hline 4 weeks & $42.7(37.2$ to 48.1$)$ & 38.3 (31.1 to 45.6$)$ & 0.716 \\
\hline 6 weeks & $45.2(40.3$ to 50.0$)$ & 41.6 (33.5 to 49.7$)$ & 0.800 \\
\hline \multicolumn{4}{|c|}{ Pain at rest (VAS, $0-10)$} \\
\hline 1 week & $-0.4(+0.8$ to -1.5$)$ & $-1.3(-0.6$ to -2.1$)$ & 0.375 \\
\hline 2 weeks & $-1.1(+0.2$ to -2.3$)$ & $-1.9(-0.8$ to -3.0$)$ & 0.534 \\
\hline 4 weeks & $-1.6(-0.3$ to -2.9$)$ & $-2.1(-0.8$ to -3.4$)$ & 0.668 \\
\hline 6 weeks & $-1.8(-0.6$ to -2.9$)$ & $-2.2(-0.9$ to -3.4$)$ & 0.705 \\
\hline
\end{tabular}

Pain during usual activities (VAS, 0-10)

$$
\begin{array}{llll}
1 \text { week } & -2.1(-0.6 \text { to }-3.6) & -2.3(-1.3 \text { to }-3.3) & 0.382 \\
2 \text { weeks } & -2.8(-1.5 \text { to }-4.2) & -3.4(-2.4 \text { to }-4.4) & 0.490 \\
4 \text { weeks } & -3.8(-2.4 \text { to }-5.2) & -4.1(-2.9 \text { to }-5.3) & 0.682 \\
6 \text { weeks } & -4.1(-2.7 \text { to }-5.6) & -4.4(-3.2 \text { to }-5.6) & 0.774
\end{array}
$$

\begin{tabular}{|c|c|c|c|}
\hline 1 week & $-0.69(-0.05$ to -1.34$)$ & $-0.64(+0.11$ to -1.38$)$ & 0.496 \\
\hline 2 weeks & $-1.06(-0.29$ to -1.83$)$ & $-0.56(+0.28$ to -1.40$)$ & 0.508 \\
\hline 4 weeks & $-1.24(-0.29$ to -2.18$)$ & $-0.93(-0.19$ to -1.67$)$ & 0.532 \\
\hline 6 weeks & $-1.15(-0.20$ to -2.09$)$ & $-0.76(-0.05$ to -1.47$)$ & 0.519 \\
\hline \multicolumn{4}{|c|}{ Oorsiflexion ROM (WBLT, cm) } \\
\hline 1 week & $2.4(-0.1$ to 4.9$)$ & $3.6(1.1$ to 6.0$)$ & 0.414 \\
\hline 2 weeks & 4.1 (1.9 to 6.3$)$ & $4.8(2.2$ to 7.4$)$ & 0.550 \\
\hline 4 weeks & $5.2(3.0$ to 7.4$)$ & 5.9 (3.2 to 8.6$)$ & 0.643 \\
\hline 6 weeks & 5.7 (3.4 to 8.0 ) & 5.9 (3.1 to 8.7$)$ & 0.668 \\
\hline
\end{tabular}

Oedema (Figure-of-8, cm)

${ }^{a}$ Relative treatment effect (nparLD analysis), for appreciation of the time main effect. Corresponds to an effect size of the time. Values range [0, 1], relative to the null hypothesis $(\mathrm{H} 0)$ expected value $(0,5)$

LEFS Lower Extremity Functional Scale, NCS neurocryostimulation, ROM range of motion, RTE relative treatment effect, VAS visual analog scale, WBLT WeightBearing Lunge Test. 


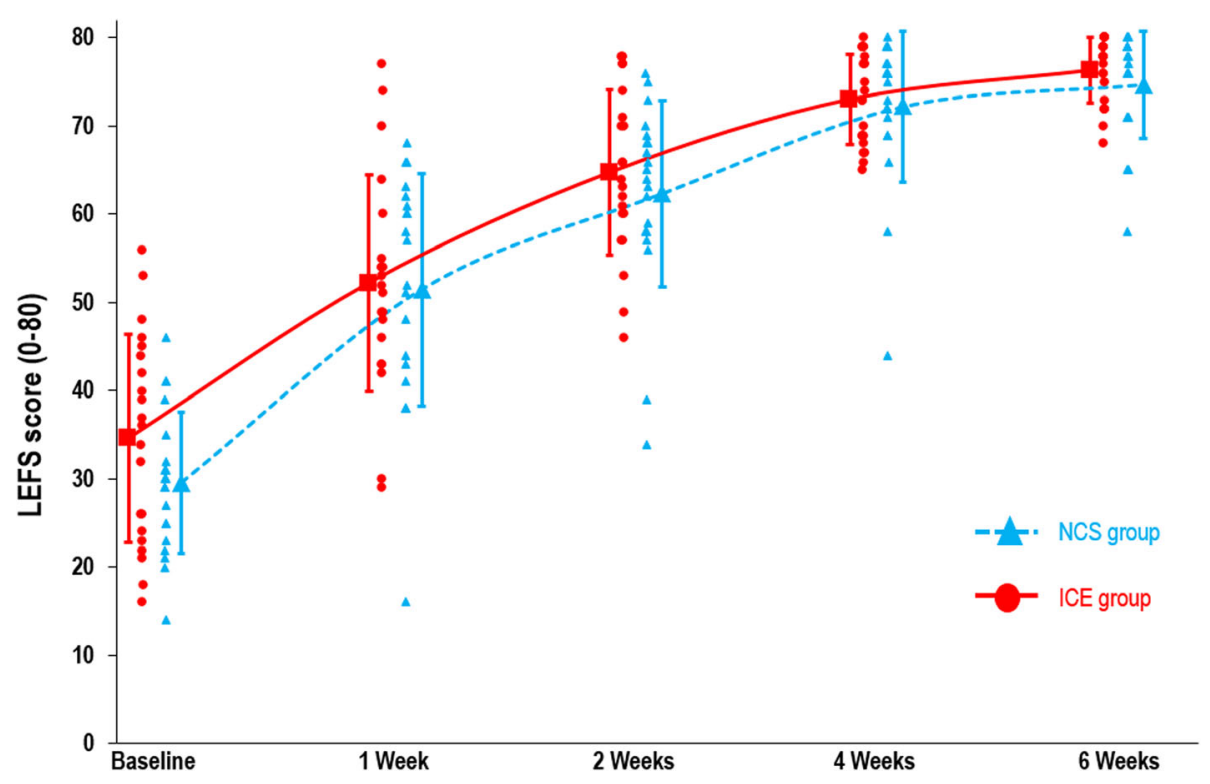

Fig. 3 LEFS Scores. Lower Extremity Functional Scale (LEFS) mean scores (larger markers) for NCS group ( $n=20)$ and ice group ( $n=21)$, and individual scores (smaller markers). Higher scores indicate better function. Error bars show standard deviation (SD) of the means. NCS: neurocryostimulation

packs and cryocuffs) on pain, knee mobility and edema seven days after total knee replacement surgery[17]. Of note, the NCS treatment protocol in Demoulin et al.'s study resulted in cooling of the skin to an average temperature of $14^{\circ} \mathrm{C}$, which is warmer than the temperatures recommended to expect benefits from cryotherapy[40, 41].
This methodological limitation regarding the level of cooling is one important reason why the use of cold in injury care is often questioned [11, 12]. Cryotherapy has been proposed to control pain by slowing nerve conduction and to decrease secondary ischemic or enzymatic tissue death [42]. Demonstrated effects of cryotherapy include significant cutaneous analgesia below $13.6^{\circ} \mathrm{C}$, a

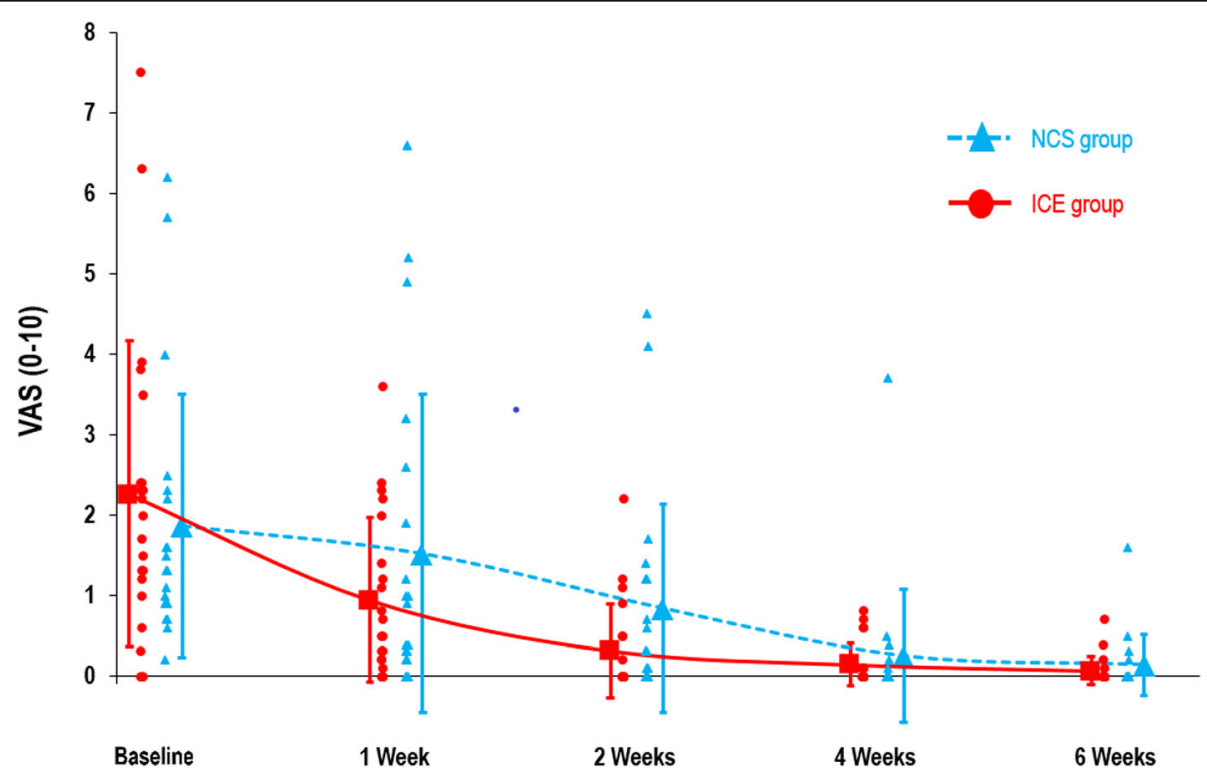

Fig. 4 Pain at Rest Scores. Pain intensity at rest during the last $48 \mathrm{~h}$. Data show mean scores (larger markers) for NCS group $(n=20)$ and ice group $(n=21)$, and individual scores (smaller markers). Error bars show standard deviation (SD) of the means. NCS: neurocryostimulation; VAS: visual analog scale 


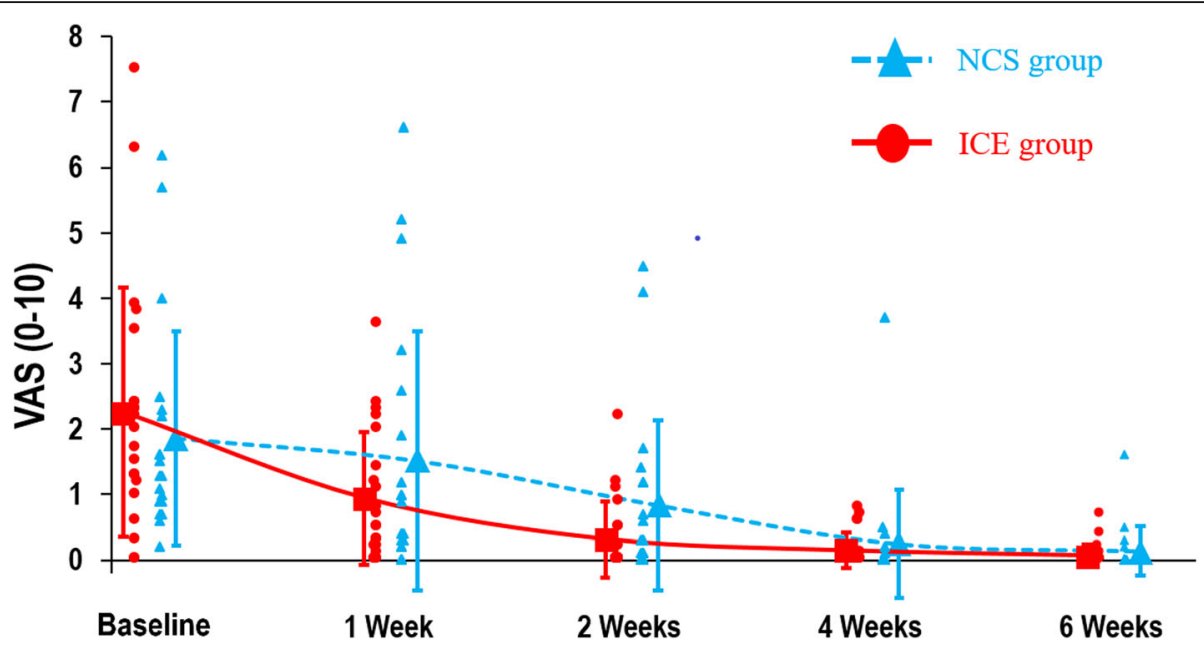

Fig. 5 Pain during Usual Activities Scores. Pain intensity during usual activities for the last $48 \mathrm{~h}$. Data show mean scores (larger markers) for NCS group ( $n=20)$ and ice group $(n=21)$, and individual scores (smaller markers). Error bars show standard deviation (SD) of the means. NCS: neurocryostimulation; VAS: visual analog scale

$10 \%$ decrease in nerve conduction below $12.5^{\circ} \mathrm{C}$, and a $50 \%$ decrease in local metabolism below $11^{\circ} \mathrm{C}[40,41]$. In numerous studies looking at the effects of cryotherapy, those goal temperatures have not been reached, [13] contributing to the persistent difficulty in clarifying the clinical usefulness of cryotherapy in health care. In fact, the achieved temperature depends on many factors, such as the cryotherapy method used, the duration of application, the initial skin temperature and the thickness of the subcutaneous fatty tissue, among others. In our study, the skin temperature attained following the application of ice did not reach the mentioned thresholds, while it did reach these thresholds following NCS.

Mourot et al. demonstrated that after NCS, the rate of rewarming during the first minutes is quicker than after ice application, and that skin temperature is no longer significantly different between groups after one [15] or six minutes [14]. This could suggest that the level of deep tissue induced cooling would be similar between NCS and ice, and that the additional cooling induced by NCS would be limited to the superficial tissues. In terms of potential analgesic and metabolic slowing effects in deep injured tissues, such as ligaments in the case of sprains, NCS and ice therefore likely have similar effects, and the effects on the autonomic nervous system attributed to NCS, the so-called "thermal shock", may result from a greater or quicker cooling of the surface structures only, and not of the deep structures.

Although there is no evidence of the clinical effectiveness of NCS, its physiological effects on circulatory parameters have been described. Mourot et al. observed rapid systemic cutaneous vasoconstriction and increased blood pressure in response to NCS application in healthy subjects, $[14,15]$ which are reactions comparable to those observed when evaluating the effect of hand immersion in cold water (cold pressor test )[43]; they did not, however, see those reactions with ice application $[14,15]$. This response, referred to as thermal shock and involving the autonomic nervous system, is the basis of the hypothesis that NCS holds potential as a treatment modality. In the current study, rapid and significantly greater decreases in skin temperature were observed in the NCS group compared to the ice group, suggesting that a thermal shock was generally obtained in the former group. That did not, however, translate into the greater therapeutic benefits that were expected.

From a more practical point of view, the use of NCS requires a substantial investment of money at the time of purchase, in addition to the cost of periodic refilling of the gas cylinder and eventual maintenance of the unit. It is therefore more expensive than the use of ice, and also has the disadvantage of not being easily transportable for use at home, or on the sports field, for examples. There are smaller models of NCS devices designed to be transported, but those were not used in this study. NCS nevertheless holds a distinct advantage over ice application: with an application time of less than two minutes, it is much quicker for the patient than the ten, fifteen or even twenty minute application period recommended when using ice $[13,44]$.

When interpreting the results of this study, some limitations should be kept in mind, the main one being that there was no control group, i.e. a group not receiving cryotherapy of any kind. Including such a group would have made it possible to study the effects of both NCS and ice. This study also did not control for placebo 
effect, which would have required creating a treatment similar to NCS, but with no effects related to cooling of the skin; however, a credible sham NCS that would feel cold enough without actually lowering skin temperature significantly seemed impossible to put in place. Regarding treatment frequency, optimal NCS application parameters are unknown. Here, treatments were offered at a frequency of three times per week for the first two weeks, and once per week for the following two weeks. These parameters were chosen because they were considered a good compromise between the desire to administer numerous treatments in the very acute phase of the injury, and the logistical challenges that are intrinsic to delivering outpatient physiotherapy treatments, such as scheduling and travel time.

Although physiotherapists were given specific guidelines for choosing which interventions to use for the rehabilitation program, the exact parameters for interventions other than cryotherapy were neither imposed nor recorded. This is a potential source of variation between participants that could have affected the results. Also, the protocol for the ice group included elevation of the lower extremity, with ice applied all around the ankle with bags of crushed ice that also applied compression. The potential effects of ice cannot therefore be isolated from those of compression and elevation. Finally, the age of the participants, with a mean of 28 years (SD: 9), characterizes a relatively young sample of patients, which may be a limitation to the overall generalizability of the findings.

Conversely, one of the greatest strengths of this study was the ability to include participants quickly, as evidenced by the average of 2.8 days between the injury and the first treatment received. Another strength is the study design which made it possible to evaluate the progression of patients during the phase of intense metabolic and inflammatory activity (1-week and 2-weeks follow-ups). Also, the near-perfect retention rate is worth mentioning.

\section{Conclusion}

The results of this RCT suggest that a more sophisticated and costly application of cryotherapy, namely neurocryostimulation, is no more effective than the traditional application of ice in improving functional recovery, pain, edema and ankle dorsiflexion ROM during the first six weeks of physiotherapy treatments in individuals with an acute lateral ankle sprain. Other RCTs would be useful to better evaluate the potential utility or superiority of this treatment modality by including different populations, injuries and pathologies, varying NCS application protocols, and including a proper control group not receiving any cryotherapy. Altogether, current evidence suggests that the only advantage of
NCS compared to ice is the rapidity with which it is applied, which may be a key element to consider in a busy high-volume rehabilitation practice.

\begin{abstract}
Abbreviations
Cl: Confidence interval; LAS: Lateral ankle sprain; LEFS: Lower extremity functional scale; MCID: Minimal clinically important difference; MDC: Minimal detectable change; MRI: Magnetic resonance imaging;

NCS: Neurocryostimulation; nparLD: Nonparametric analysis for longitudinal data; RCT: Randomized clinical trial; RICE: Rest, ice, compression, elevation; ROM: Range of motion; SD: Standard deviation; SEM: Standard error of measurement; VAS: Visual analog scale; WBLT: Weight-bearing lunge test; $\bar{X}:$ Mean
\end{abstract}

\section{Acknowledgements}

The authors would like to thank all the patients who participated to this study, the physiotherapists who provided the interventions (J.-F. Brochu, S.M. Carbonneau, E. Dupont) and Jean Leblond for support in statistical analyses.

\section{Authors' contributions}

JT contributed to protocol development, managed the implementation of the clinical trial, collected the data, and drafted the article. JSR was the main contributor to study design and protocol development, data analysis and interpretation, and contributed to the review of the article. LJH contributed to study design and protocol development, data interpretation, and review of the article. All authors read and approved the final manuscript.

\section{Funding}

The project was funded by grants from Cryofos Medical GmbH, Atlas Medic, and the authors' research centre (Center for Interdisciplinary Research in Rehabilitation and Social Integration). A scholarship was awarded to JT by the Ordre Professionnel de la Physiothérapie du Québec. JSR was supported by a salary award from the Canadian Institutes of Health Research.

\section{Availability of data and materials}

The datasets used and/or analysed during the current study are available from the corresponding author on request.

\section{Ethics approval and consent to participate}

The sectorial health sciences research ethics committee of Laval University (Quebec city, Canada) approved this study (\#2015-053). All participants signed an informed consent prior to inclusion.

\section{Consent for publication}

Not applicable.

\section{Competing interests}

The research team received funding from Cryofos Medical $\mathrm{GmbH}$, manufacturer of the NCS device used in this study, as well as from Atlas Medic, distributor of Cryofos products in Canada. The unit used in the study was loaned free of charge by Atlas Medic. Cryofos Medical GmbH and AtlasMedic were consulted in regard to the study design prior to beginning of recruitment (mainly for NCS application protocols). They were not involved (nor were any other funding body) in data collection, analysis, and interpretation, in the writing of the manuscript or in the decision to submit for publication.

The authors declare that they have no other apparent or real conflict of interest.

\section{Author details}

'Department of Rehabilitation, Faculty of Medicine, Université Laval, Quebec City, Quebec, Canada. ${ }^{2}$ Center for Interdisciplinary Research in Rehabilitation and Social Integration, Quebec Rehabilitation Institute, Quebec City, Quebec, Canada. ${ }^{3}$ Department of Radiology and Nuclear Medicine, Faculty of Medicine, Université Laval, Quebec City, Quebec, Canada. 
Received: 17 June 2020 Accepted: 16 November 2020

Published online: 01 December 2020

\section{References}

1. van den Bekerom MP, Kerkhoffs GM, McCollum GA, Calder JD, van Dijk CN Management of acute lateral ankle ligament injury in the athlete. Knee Surg Sports Traumatol Arthrosc. 2013;21(6):1390-5.

2. Medical M's. Nursing \& Allied Health Dictionary. 5th edition ed. St-Louis, MO: Mosby; 2005

3. Doherty C, Delahunt E, Caulfield B, Hertel J, Ryan J, Bleakley C. The incidence and prevalence of ankle sprain injury: a systematic review and meta-analysis of prospective epidemiological studies. Sports Med. Jan 2014; 44(1):123-40.

4. Verhagen EA, van Mechelen W, de Vente W. The effect of preventive measures on the incidence of ankle sprains. Clin J Sport Med. 2000;10(4): 291-6.

5. Kerkhoffs GM, van den Bekerom M, Elders LA, et al. Diagnosis, treatment and prevention of ankle sprains: an evidence-based clinical guideline. $\mathrm{Br} J$ Sports Med. 2012;46(12):854-60.

6. Kaminski TW, Hertel J, Amendola N, et al. National Athletic Trainers' association position statement: conservative management and prevention of ankle sprains in athletes. J Athl Train. 2013:48(4):528-45.

7. van den Bekerom MP, Struijs PA, Blankevoort $L$, Welling $L$, van Dijk $C N$ Kerkhoffs GM. What is the evidence for rest, ice, compression, and elevation therapy in the treatment of ankle sprains in adults? J Athl Train. 2012;47(4): 435-43.

8. Vuurberg G, Hoorntje A, Wink LM, et al. Diagnosis, treatment and prevention of ankle sprains: update of an evidence-based clinical guideline. Br J Sports Med. 2018.

9. Tiemstra JD. Update on acute ankle sprains. Am Fam Physician. 2012;85(12): 1170-6.

10. Chaudhry H, Simunovic N, Petrisor B. Cochrane in CORR (R): surgical versus conservative treatment for acute injuries of the lateral ligament complex of the ankle in adults (review). Clin Orthop Relat Res. 2015;473(1):17-22.

11. Doherty C, Bleakley C, Delahunt E, Holden S. Treatment and prevention of acute and recurrent ankle sprain: an overview of systematic reviews with meta-analysis. Br J Sports Med. 2017;51(2):113-25.

12. Bleakley C, McDonough S, MacAuley D. The use of ice in the treatment of acute soft-tissue injury: a systematic review of randomized controlled trials. Am J Sports Med. 2004;32(1):251-61.

13. MacAuley D. Ice therapy: how good is the evidence? Int J Sports Med. 2001; 22:379-84.

14. Mourot L, Cluzeau C, Regnard J. Hyperbaric gaseous cryotherapy: effects on skin temperature and systemic vasoconstriction. Arch Phys Med Rehabil. 2007;88(10):1339-43.

15. Mourot L, Cluzeau C, Regnard J. Physiological assessment of a gaseous cryotherapy device: thermal effects and changes in cardiovascular autonomic control. Ann Readapt Med Phys. 2007:50(4):209-17.

16. Chatap G, De Sousa A, Giraud K, Vincent JP. Acute pain in the elderly study $\mathrm{G}$. pain in the elderly: prospective study of hyperbaric $\mathrm{CO} 2$ cryotherapy (neurocryostimulation). Joint Bone Spine. 2007;74(6):617-21.

17. Demoulin C, Brouwers M, Darot S, Gillet P, Crielaard JM, Vanderthommen M Comparison of gaseous cryotherapy with more traditional forms of cryotherapy following total knee arthroplasty. Ann Phys Rehabil Med. 2012; 55(4):229-40.

18. Sman AD, Hiller CE, Refshauge KM. Diagnostic accuracy of clinical tests for diagnosis of ankle syndesmosis injury: a systematic review. Br J Sports Med. 2013;47(10):620-8

19. Schwieterman B, Haas D, Columber K, Knupp D, Cook C. Diagnostic accuracy of physical examination tests of the ankle/foot complex: a systematic review. Int J Sports Phys Therapy. 2013;8(4):416-26.

20. Lau BC, Moore LK, Thuillier DU. Evaluation and Management of Lateral Ankle Pain Following Injury. JBJS reviews. 2018;6(8):e7.

21. Lynch SA. Assessment of the injured ankle in the athlete. J Athl Train. 2002; 37(4):406-12.

22. Wiebking U, Pacha TO, Jagodzinski M. An accuracy evaluation of clinical, arthrometric, and stress-sonographic acute ankle instability examinations Foot Ankle Surg. 2015;21(1):42-8.

23. Stiell IG, Greenberg GH, McKnight RD, Nair RC, McDowell I, Worthington JR. A study to develop clinical decision rules for the use of radiography in acute ankle injuries. Ann Emerg Med. 1992/04/01/. 1992;21(4):384-90.
24. Binkley J, Stratford P, Lott S, Riddle D. The lower extremity functional scale (LEFS) scale development, measurement properties, and clinical application. Phys Ther. 1999;79:371-83.

25. Alcock GK, Stratford PW. Validation of the lower extremity functional scale on athletic subjects with ankle sprains. Physiother Can. 2002;(Fall 2002):23340.

26. Pan S-L, Liang H-W, Hou W-H, Yeh T-S. Responsiveness of SF-36 and Lower Extremity Functional Scale for assessing outcomes in traumatic injuries of lower extremities. Injury. 2014;45(11):1759-63.

27. Mehta SP, Fulton A, Quach C, Thistle M, Toledo C, Evans NA. Measurement properties of the lower extremity functional scale: a systematic review. J Orthop Sports Phys Ther. 2016;46(3):200-16.

28. Rene F, Casimiro L, Tremblay $M$, et al. [Fiabilité test retest et validité de construit de la version française de L'Échelle fonctionnelle des membres inférieurs (ÉFMI), partie II.]. Physiotherapy Canada. Physiotherapie Canada Spring. 2011;63(2):249-55.

29. Rene F, Casimiro L, Tremblay M, et al. [Une version canadienne française du Lower Extremity Functional Scale (LEFS): L'Échelle fonctionnelle des membres inférieurs (ÉFMI), partie I.]. Physiotherapy Canada. Physiotherapie Canada Spring. 2011;63(2):242-8.

30. Katz J, Melzack R. Measurement of pain. Surg Clin North Am. 1999;79(2): 231-52.

31. Gallagher EJ, Liebman M, Bijur PE. Prospective validation of clinically important changes in pain severity measured on a visual analog scale. Ann Emerg Med. 2001;38(6):633-8.

32. Mawdsley RH, Hoy DK, Erwin PM. Criterion-related validity of the figure-ofeight method of measuring ankle edema. J Orthop Sports Phys Ther. 2000; 30(3):149-53.

33. Peterson EJ, Irish SM, Lyons CL, et al. Reliability of Water Volumetry and the Figure of Eight Method on Subjects With Ankle Joint Swelling. J Orthopaedic Sports Physical Therapy. 1999/10/01. 1999;29(10):609-15.

34. Tatro-Adams D, McGann SF, Carbone W. Reliability of the figure-of-eight method of ankle measurement. J Orthop Sports Phys Ther. 1995;22(4):161-3.

35. Rohner-Spengler M, Mannion A, Babst R. Reliability and minimal detectable change for the figure-of-eight-20 method of measurement of ankle edema. J Orthop Sports Phys Ther. 2007;37:199-205.

36. Powden CJ, Hoch JM, Hoch MC. Reliability and minimal detectable change of the weight-bearing lunge test: A systematic review. Man Ther. 2015.

37. Petersen W, Rembitzki IV, Koppenburg AG, et al. Treatment of acute ankle ligament injuries: a systematic review. Arch Orthop Trauma Surg. 2013; 133(8):1129-41.

38. Noguchi K, Gel Y, Brunner E, Konietschke F. nparLD: an R software package for the nonparametric analysis of longitudinal data in factorial experiments. J Stat Softw. 2012;50(12):1-23.

39. Richer N, Marchand AA, Descarreaux M. Management of Chronic Latera Epicondylitis with Manual Therapy and Local Cryostimulation: a pilot study. J Chiropractic Med. 2017;16(4):279-88.

40. McMeeken J, Lewis MM, Cocks S. Effects of cooling with simulated ice on skin temperature and nerve conduction velocity. Aust J Physiother. 1984; 30(4):111-4.

41. Zachariassen KE. Hypothermia and cellular physiology. Arctic Med Res. 1991; 50(Suppl 6):13-7.

42. Hubbard TJ, Denegar CR. Does Cryotherapy improve outcomes with soft tissue injury? J Athl Train. 2004;39(3):278-9.

43. Pouwels S, Van Genderen ME, Kreeftenberg HG, et al. Utility of the cold pressor test to predict future cardiovascular events. Expert Rev Cardiovasc Ther. 2019:17(4):305-18.

44. Bleakley C, McDonough S, MacAuley D. Cryotherapy for acute ankle sprains: a randomised controlled study of two different icing protocols. Br J Sports Med. 2006:40:700-5.

\section{Publisher's Note}

Springer Nature remains neutral with regard to jurisdictional claims in published maps and institutional affiliations. 